\title{
CONTROLE PREDITIVO APLICADO A UMA PLANTA PILOTO DE NEUTRALIZAÇÃO DE PH
}

\author{
CHRISTIAM MORALES ${ }^{1}$, Luís LOURENÇO ${ }^{1}$, CLAUDio GARCIA ${ }^{1}$ \\ 1. Laboratório de Automação e Controle, Departamento de Engenharia de Telecomunicações e Controle, \\ Universidade de São Paulo \\ CEP 05508900, São Paulo, Brasil \\ E-mails: cmoralesa@usp.br, Ifnlourenco@gmail.com, clgarcia@lac.usp.br
}

\begin{abstract}
Resumo- Neste trabalho é implementado um controlador preditivo baseado em modelo (MPC), em uma planta piloto de neutralização de $\mathrm{pH}$, para o controle das variáveis do processo. Na etapa de identificação do processo é aplicado o algoritmo EMPEM para estimar os parâmetros dos modelos de processo, a fim de ser utilizados no controlador preditivo proposto. Os sinais de controle que ingressarão no processo são calculados utilizando o algoritmo QDMC (Quadratic Dynamic Matrix Control). Finalmente, o controlador desenvolvido é testado em modo servo.
\end{abstract}

Palavras-chave—Controle preditivo, Algoritmo de controle QDMC, Identificação de Sistemas, Controle de $\mathrm{pH}$.

\section{Introdução}

$\mathrm{O}$ processo de neutralização de $\mathrm{pH}$ pode ser encontrado em diferentes processos industriais, por exemplo tratamento de efluentes, processos biotecnológicos e processos químicos. A neutralização tem a finalidade de mudar o $\mathrm{pH}$ da vazão de entrada (influente) adicionando um reagente (variável manipulada) para obter, na vazão de saída, o pH desejado (Shinskey, 1996).

As muitas pesquisas sobre o controle do processo de neutralização de $\mathrm{pH}$ são justificadas pela alta nãolinearidade e pelo tempo morto que o processo apresenta. Por isto, diversos trabalhos propõem diferentes soluções para o controle de neutralização do pH. Em (Proudfoot et al., 1983) é aplicado um controlador convencional do tipo PI em um processo de tratamento de efluentes. Redes neuronais e controle robusto são propostos em (Sean, 1999) e (Shabani et al., 2010), respectivamente, para melhorar o controle do processo de $\mathrm{pH}$.

Neste trabalho é desenvolvido o sistema de controle utilizando uma técnica de controle avançado do tipo MPC. A principal vantagem do MPC consiste na capacidade de tratar restrições nas entradas e saídas do processo, a fim de se obter uma maior segurança de operação (Maciejowski, 2002). Os objetivos principais deste artigo são: (a) empregar o algoritmo EMPEM para estimar modelos matemáticos que permitam representar o comportamento dinâmico do processo de neutralização do $\mathrm{pH}$ e (b) implementar um controlador preditivo MPC do tipo QDMC, a fim de calcular os sinais de controle baseado nos modelos obtidos.

Este artigo está organizado da seguinte forma. Na seção 2 se descreve a planta piloto de neutralização de $\mathrm{pH}$ localizada no Laboratório de Controle de Processos Industrias da Escola Politécnica da Universidade de São Paulo. Na seção 3 é descrito o procedimento da identificação de sistemas para o processo de neutralização do $\mathrm{pH}$. Na seção 4 é apresentada a descrição de um controlador MPC e na seção 5 são apresentados os resultados experimentais. Finalmente, na seção 6 são resumidas as principais conclusões deste trabalho.

\section{Planta piloto de neutralização de $\mathbf{p H}$}

De acordo com o digrama (P\&ID) mostrado na Figura 1, a planta piloto é composta da seguinte forma:

- Um tanque de água filtrada (TF), ou um tanque de água destilada (TD) para fornecer de água ao processo.

- Um tanque de ácido principal (TAP) onde é preparada a solução de ácido clorídrico $(\mathrm{HCl})$. Esta solução de $\mathrm{HCl}$ é armazenada em um tanque intermediário (TAPI) para manter a vazão constante que ingressa no reator.

- Um tanque para preparar a solução de base (TBB) de hidróxido de sódio $(\mathrm{NaOH})$ para neutralizar a vazão de entrada ao reator através de uma bomba dosadora.

- Um tanque reator onde é realizado o processo de neutralização. Para homogeneizar a mistura é usado um agitador mecânico. $\mathrm{O}$ reator possui um eletrodo de $\mathrm{pH}$ e um transmissor de pressão diferencial para a leitura das variáveis do processo do nível e $\mathrm{pH}$ do reator. 


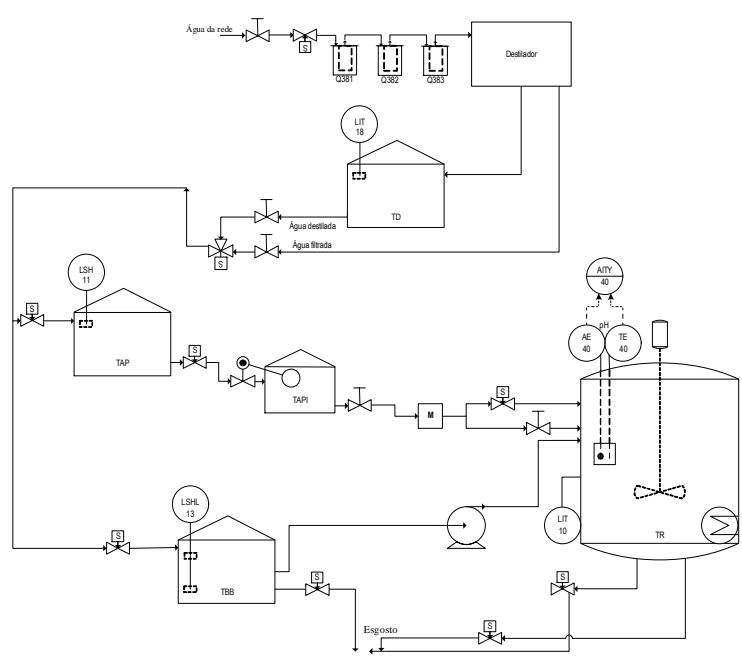

Figura 1: P\&ID da planta piloto de neutralização de $\mathrm{pH}$.

\section{Identificação do processo de neutralização de pH}

\subsection{Pré-teste}

Nesta etapa, sinais do tipo degrau são aplicados ao processo de neutralização de $\mathrm{pH}$, com a finalidade de obter parâmetros relevantes do processo, como o tempo de acomodação para cada malha.

Para isso, são realizadas variações no sinal de entrada para as malhas do nível e $\mathrm{pH}$ da seguinte forma:

- Para a malha do nível, o processo em estado estacionário possui um valor de $65 \%$ e as variações no set point são realizadas na faixa de $\pm 3 \%$.

- Para a malha do $\mathrm{pH}$, seu valor no estado estacionário é 7 e as variações no set point são realizadas no intervalo de \pm 0.5 .

onde cada patamar possui um tempo de 30 minutos. Com os dados coletados de entrada e saída, estimamse modelos de primeira ordem com tempo morto mostrados na equação (1).

$$
\begin{gathered}
\text { Nível }_{M F}=\frac{0.006472}{s+0.006} e^{-30 s} \\
p H_{M F}=\frac{0.01324}{s+0.01283} e^{-10 s}
\end{gathered}
$$

De acordo com a equação (1), Nível $l_{M F}$ e $p H_{M F}$ são modelos em malha fechada obtidos para as malhas do nível e $\mathrm{pH}$, respectivamente, incluindo os controladores PID do processo.

\subsection{Projeto do sinal de excitação}

Neste trabalho é utilizado o sinal de excitação GBN (Generalized Binary Noise), proposto por Tulleken (1990), para ser aplicado ao processo de neutralização de $\mathrm{pH}$. No desenvolvimento deste sinal é necessário calcular um vetor aleatório em função do o intervalo de amostragem $T_{a}$, o tempo de acomodação $T_{S}$, a semente e o tempo mínimo de chaveamento $T_{\text {min }}$ do sinal GBN. O tempo de acomodação é calculado aplicando um degrau aos modelos obtidos na equação (1).

A Figura 2 mostra o sinal GBN para as malhas de nível e de $\mathrm{pH}$. Este sinal foi criado com os seguintes parâmetros:

- Para a malha de nível, aplicando um degrau na função de transferência $N$ ível $l_{M F}$ é obtido um tempo de acomodação igual a 702 segundos. De acordo com (Zhu, 2001), para calcular o tempo de amostragem é assumida uma relação $T_{a}=\frac{T_{s}}{50}=15$ segundos. A semente do sinal assume um valor de 13. Finalmente, o tempo mínimo de chaveamento $T_{\text {min }}$ é assumido a partir da relação $T_{\min }=4 T_{a}=60$ segundos.

- Para a malha de pH, como no caso anterior, aplicando um degrau à função de transferência $p H_{M F}$ é obtido um tempo de acomodação de $T_{s}=315$ segundos. Para calcular o tempo de amostragem é utilizada a mesma relação que no caso anterior assumindo um valor de $T_{a}=$ $\frac{T_{s}}{50}=7$ segundos. A semente do sinal toma um valor de 7 e o tempo mínimo de chaveamento é $T_{\text {min }}=4 T_{a}=28$ segundos.
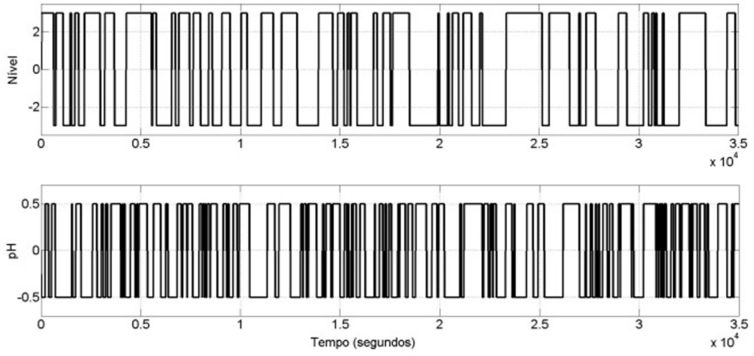

Figura 2: Sinal de excitação GBN aplicado ao processo de neutralização de $\mathrm{pH}$.

Para ambos os sinais de excitação, o fator de ajuste da frequência do sinal $n$ adota um valor de 1.5.

\subsection{Identificação em malha fechada}

Devido à instabilidade do processo, a identificação é realizada em malha fechada, isto é incluindo os controladores PID (Proporcional-Integral-Derivativo) do processo.

A estrutura dos modelos dinâmicos do processo e de perturbação adotada é representada pela equação (2):

$$
\begin{aligned}
& G(q, \theta)=\frac{B(q, \theta)}{F(q, \theta)} \\
& H(q, \theta)=\frac{C(q, \theta)}{D(q, \theta)}
\end{aligned}
$$

onde $B(q, \theta), C(q, \theta), D(q, \theta)$ e $F(q, \theta)$ são os polinômios do processo e $G(q, \theta)$ e $H(q, \theta)$ representam 
as funções de transferência do modelo do processo e do modelo de perturbação, respectivamente.

Logo, com os dados coletados das variáveis manipuladas e controladas do processo, usando um tempo de amostragem de 10 segundos, são calculados os coeficientes dos polinômios aplicando o algoritmo EMPEM proposto em (Potts et al., 2012). Este algoritmo fornece modelos estáveis para aplicações com MPC dentro de um amplo horizonte de predição.

$\mathrm{Na}$ aplicação do algoritmo EMPEM, o horizonte de predição é calculado dividindo o tempo de acomodação mais alto do processo e o tempo de amostragem empregado na coleta de dados. Neste trabalho é assumido um valor de 70. Para obter a ordem ótima dos modelos de processo e perturbação é empregando o critério FPEP (Final Prediction Error for $P$-step prediction) (Potts et al, 2012).

Uma vez realizado o cálculo da ordem ótima e a identificação do processo, as Tabelas 1 e 2 mostram os modelos do processo e de perturbação obtidos pelo algoritmo EMPEM, respectivamente.

Tabela 1 Modelos do processo.

\begin{tabular}{|c|c|}
\hline \multirow{2}{*}{ Saída } & Entrada \\
\cline { 2 - 3 } Nível & $\frac{-0.1188 z^{-2}+0.04564 z^{-3}}{1-0.844 z^{-1}-0.643 z^{-2}+0.489 z^{-3}}$ \\
\hline \multirow{2}{*}{$\mathrm{pH}$} & $\frac{0.0631 z^{-3}-0.129 z^{-4}+0.0664 z^{-5}}{1-2.049 z^{-1}+0.549 z^{-2}+1.081 z^{-3}-0.582 z^{-4}}$ \\
\hline \multirow{2}{*}{ Saídas } & Entrada \\
\cline { 2 - 3 } & $\frac{0.02273 z^{-3}-0.02263 z^{-4}}{1-1.217 z^{-1}-0.553 z^{-2}+0.769 z^{-3}}$ \\
\hline \multirow{2}{*}{ Nível } & $\frac{0.2127 z^{-2}-0.3827 z^{-3}+0.1816 z^{-4}}{214 z^{-1}+3.802 z^{-2}-1.93 z^{-3}+0.343 z^{-4}}$ \\
\hline \multirow{2}{*}{$\mathrm{pH}$} & $\frac{1-3.214 z^{-1} \text { controle do pH }}{2}$
\end{tabular}

Tabela 2 Modelos de perturbação.

\begin{tabular}{|c|c|}
\hline \multirow{2}{*}{ Saída } & Entrada \\
\cline { 2 - 2 } & Distúrbio de processo \\
\hline Nível & $\frac{1+0.1382 z^{-1}}{1-0.474 z^{-1}-0.283 z^{-2}-0.00992 z^{-3}-0.0442 z^{-4}-0.177 z^{-5}}$ \\
\hline $\mathrm{pH}$ & $\frac{1-0.0146 z^{-1}-0.3227 z^{-2}+0.1352 z^{-3}}{1-0.82 z^{-1}-0.505 z^{-2}+0.36 z^{-3}-0.0259 z^{-4}-0.0081 z^{-5}}$ \\
\hline
\end{tabular}

\subsection{Validação dos modelos}

A etapa de validação consiste em avaliar o índice fit calculado através dos dados reais de saída do processo $(y(t))$ e os dados das saídas estimados pelos modelos $(\hat{y}(t))$, como é mostrado na equação (3).

$$
f i t(\%)=100 \cdot\left(1-\frac{\|y(t)-\hat{y}(t)\|}{\|y(t)-\bar{y}(t)\|}\right)
$$

Os índices fit obtidos foram calculados realizando uma autovalidação tal como é mostrado na Figura 3.

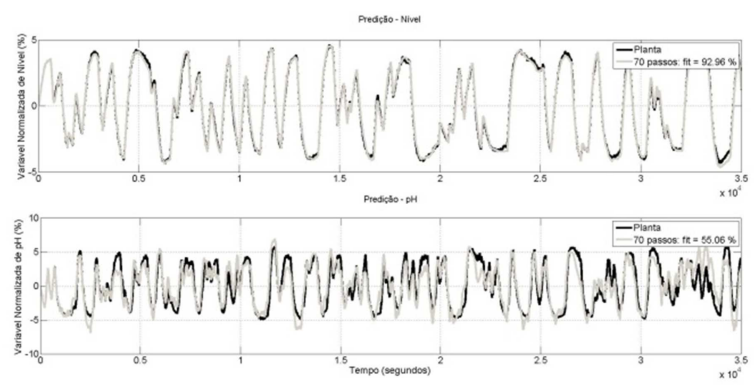

Figura 3: Autovalidação para uma predição de 70 passos à frente.

De acordo com os resultados mostrados na Figura 3, o algoritmo EMPEM apresenta um melhor índice fit para o nível. No caso do $\mathrm{pH}$, se obtêm um índice fit baixo, isto devido à não-linearidade do sistema, mas observa-se uma boa coerência com a saída do processo.

\section{Controle preditivo baseado em modelo}

O controle preditivo baseado em modelo MPC (Model Predictive Control) é um algoritmo de controle que permite calcular os sinais de entrada que ingressam no processo baseado em um modelo linear. O principal objetivo do MPC é otimizar o processo dentro de um horizonte de predição, restringindo as variáveis de entrada e saída do processo (Camacho; Bordons, 2000).

Um tipo de MPC muito empregado na indústria é o algoritmo QDMC. O algoritmo QDMC proposto em (García; Morshedi, 1986) emprega um modelo de resposta ao degrau para predizer as respostas futuras e calcular o sinal de entrada do processo. Para calcular os sinais de controle é necessário, em cada tempo de amostragem, minimizar uma função custo quadrática on-line, esta ultima é representada através da equação (4).

$$
\begin{gathered}
J={ }_{\Delta \mathrm{u}}^{\min } \sum_{i=1}^{P} \delta\left(y^{r e f}(k+i)-\hat{y}(k+i)\right)^{2}+ \\
\sum_{i=0}^{c-1} \lambda \Delta u(k+i)^{2}
\end{gathered}
$$

sujeito a:

$$
\begin{gathered}
\Delta u_{\min } \leq \Delta u \leq \Delta u_{\max } \\
u_{\min } \leq u \leq u_{\max } \\
y_{\min } \leq y \leq y_{\max }
\end{gathered}
$$

De acordo com a equação (4), $P$ é o horizonte de predição, c é o horizonte de controle e $\delta$ e $\lambda$ são os pesos da saída e a variável manipulada. Na equação (5), $\Delta u_{\min }$ e $\Delta u_{\max }$ são os valores máximos e mínimos da variação na entrada; $u_{\text {min }}$ e $u_{\max }$ são os limites máximos e mínimos do valor atual da entrada; $y_{\min }$ e $y_{\max }$ são os limites máximos e mínimos do valor atual da saída.

No caso de processos multivariáveis, a função custo apresenta a mesma forma, mas $\delta$ e $\lambda$ são matrizes diagonais representadas por $\mathbf{Q}$ e $\mathbf{R}$, respectivamente. 


\subsection{Esquema de controle do algoritmo QDMC}

Para este caso, o algoritmo QDMC envia o valor dos sinais de controle ótimos ao processo de neutralização de pH, como é mostrado na Figura 4.

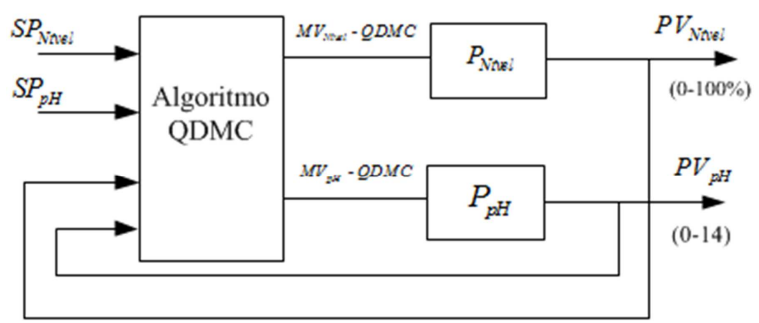

Figura 4: Esquema de controle utilizando o algoritmo QDMC.

Os parâmetros de sintonia e as restrições empregadas no algoritmo QDMC, para as malhas de nível e de $\mathrm{pH}$, que foram obtidas de maneira experimental, são mostrados na Tabela 3. O horizonte de predição e o horizonte de controle adotado são de 70 e 2 , respectivamente.

Tabela 3 Parâmetros de sintonia e restrições do algoritmo QDMC.

\begin{tabular}{|c|c|c|}
\hline $\begin{array}{c}\text { Pesos e Restrições } \\
\text { QDMC }\end{array}$ & Nível & $\mathrm{pH}$ \\
\hline Peso na Entrada & 10 & 10 \\
\hline Peso na Saída & 1 & 0.3 \\
\hline $\begin{array}{c}\text { Valor máximo no } \\
\text { valor atual da entrada }\end{array}$ & $65 \%$ & $80 \%$ \\
\hline $\begin{array}{c}\text { Valor mínimo no valor } \\
\text { atual da entrada }\end{array}$ & $15 \%$ & $30 \%$ \\
\hline $\begin{array}{c}\text { Valor máximo no } \\
\text { valor atual da saída }\end{array}$ & $70 \%$ & 7.6 \\
\hline $\begin{array}{c}\text { Valor mínimo no valor } \\
\text { atual da saída }\end{array}$ & $60 \%$ & 6.4 \\
\hline $\begin{array}{c}\text { Limite superior na } \\
\text { variação da entrada }\end{array}$ & $5 \%$ & $5 \%$ \\
\hline $\begin{array}{c}\text { Limite inferior na } \\
\text { variação da entrada }\end{array}$ & $-5 \%$ & $-5 \%$ \\
\hline
\end{tabular}

\section{Resultados experimentais}

Os modelos obtidos para as malhas do nível e $\mathrm{pH}$ da seção 3 são testados no algoritmo QDMC e comparados com a resposta obtida pelo controlador convencional PID. Esta comparação entre controladores é realizada aplicando degraus individualmente a cada variável de processo.

As respostas obtidas pelo algoritmo QMDC e o controlador PID, para a malha do nível, são mostradas nas Figuras 5 e 6 , respectivamente, e a Figura 7 mostra o sinal de controle do nível gerado pelo algoritmo QDMC. O ensaio foi realizado inserindo uma variação no set point do nível mantendo o set point do $\mathrm{pH}$ constante.

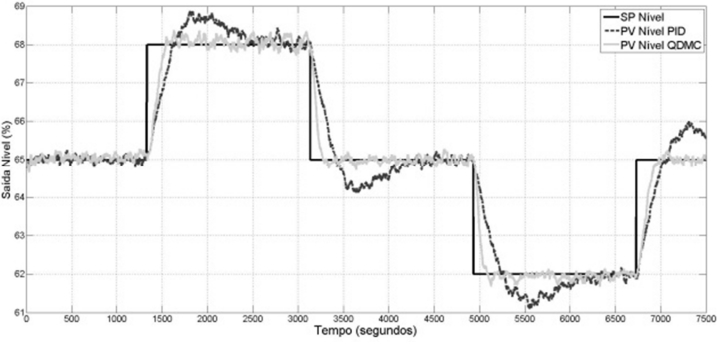

Figura 5: Comparação das respostas do processo entre os controladores PID e o QDMC insere uma variação na referencia do nível.

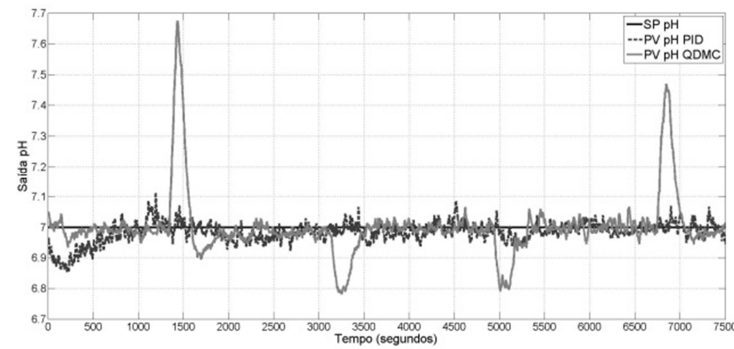

Figura 6: Comparação das respostas do processo entre os controladores PID e o QDMC mantendo a referencia do $\mathrm{pH}$ constante.

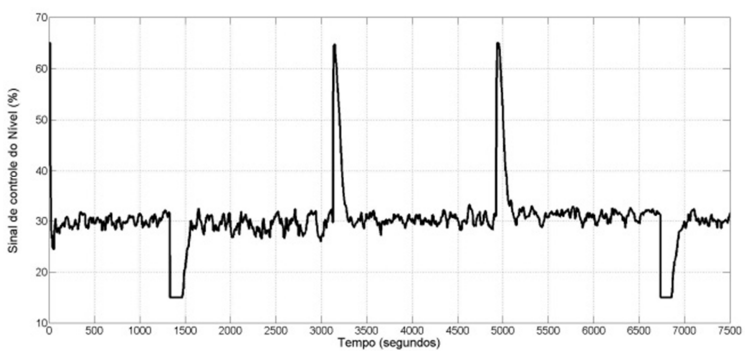

Figura 7: Sinal de controle gerado pelo algoritmo QDMC para o processo do nível.

De acordo com a Figura 5, nota-se a eficiência do algoritmo QDMC que baseado nos modelos EMPEM, permite uma melhor subida, descida e estabilidade nos diferentes pontos de operação que o controlador PID. Na Figura 6, percebe-se sobre sinal na saída do $\mathrm{pH}$ no momento que é realizada a variação do set point do nível. Isto pode acontecer, inicialmente, para compensar o aumento do acido no reator. Finalmente, na Figura 7 se mostra o sinal de controle aplicado na malha do nível se encontra dentro das restrições adotadas.

Da mesma forma é realizado o teste para a malha do $\mathrm{pH}$. As respostas obtidas pelos controladores QMDC e PID são mostradas nas Figuras 8 e 9, respectivamente. A Figura 10 mostra o sinal de controle do $\mathrm{pH}$ gerado pelo algoritmo QDMC. 


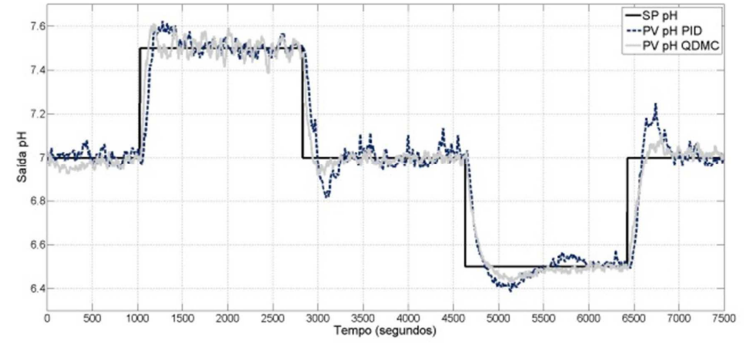

Figura 8: Comparação das respostas do processo entre os controladores PID e o QDMC, inserindo uma variação na referencia do $\mathrm{pH}$.

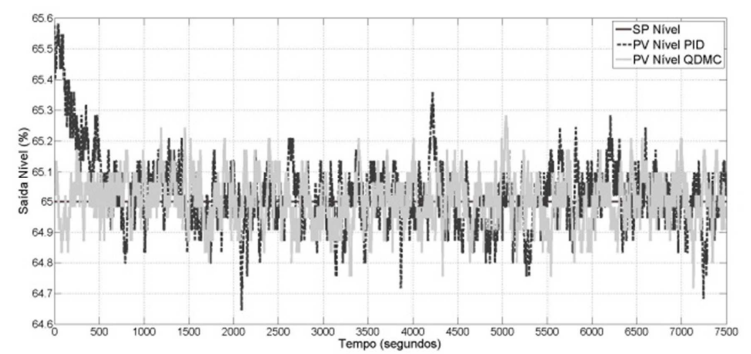

Figura 9: Comparação das respostas do processo entre os controladores PID e o QDMC mantendo a referencia do nível constante.

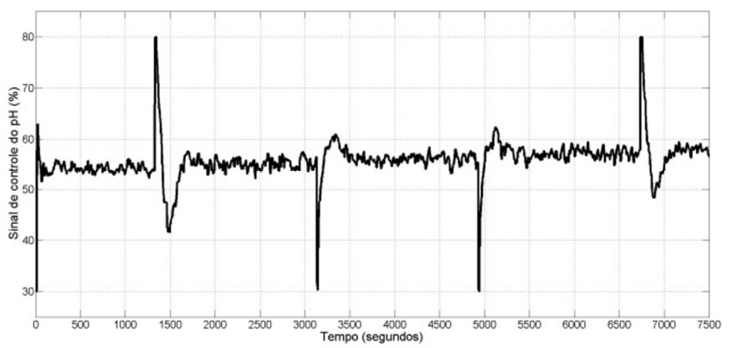

Figura 10: Sinal de controle gerado pelo algoritmo QDMC para o processo do $\mathrm{pH}$.

Conforme mostra a Figura 8, o algoritmo QDMC consegue diminuir as oscilações e reduzir o sobre sinal nos pontos de operação testados apesar das características assimétrica e à não-linearidade do processo. Na Figura 9, o nível foi mantido na faixa de $\pm 0.2 \%$ do ponto estacionário. Finalmente, na Figura 10 o sinal de controle do $\mathrm{pH}$ foi mantido dentro da faixa estabelecida.

\section{Conclusões}

Este artigo apresentou uma estratégia de controle avançado, baseado em um controlador preditivo do tipo QDMC, aplicada a uma planta de neutralização de $\mathrm{pH}$. O controlador clássico PID e o algoritmo QDMC foram comparados baseados em resultados experimentais.

De uma forma geral, os resultados obtidos demonstram a alta capacidade do algoritmo QDMC para manter estáveis as malhas processo, melhorando o desempenho do controle do processo de neutralização do $\mathrm{pH}$, tornando-o bem menos oscilatório e reduzindo o sobre sinal quando há uma variação no set point do controlador.

\section{Agradecimentos}

Os autores agradecem às agencias brasileiras CAPES e CNPq pelo financiamento.

\section{Referências Bibliográficas}

Camacho, E. F.; Bordons, C. (2000). Model Predictive Control, Springer-Verlag, London,. DOI: $10.1080 / 00986448608911397$

García, C. E.; Morshedi, A. M. (1986). Quadratic programming solution of dynamic matrix control (QDMC). Chemical Engineering Communications, 46, 73-87.

Ljung, L. (1999). System Identification: Theory for the user. Prentice Hall, Upper Saddle River, NJ, 2nd edition.

Maciejowski, J. M. (2002) . Predictive Control with Constraints, Prentice Hall, Harlow, England.

Proudfoot, C.G.; Gawthrop, P.J.; Jacobs, O.L.R. (1983). Selftuning PI control of a $\mathrm{pH}$ neutralisation process. Control Theory and Applications, IEE Proceedings D, Vol. $130 \mathrm{~N}^{\circ} 5$, pp. 267-272. DOI: 10.1049/ip-d.1983.0046

Potts, A. S.; Romano, R. A.; Garcia, C. (2012). Improving performance and stability of MRI methods in closed-loop. In: International Symposium on Advanced Control of Chemical Processes ADCHEM 2012, Singapore.

Sean, K., D. (1999). Control of $\mathrm{pH}$ in chemical processes using artificial neural networks. thesis, School of Engineering Liverpool John Moores University.

Shabani, R.; Khaki S. A.; Salahshoor K. (2010). Robust Control of a pH Neutralization Process Plant Using QFT. International Conference on Control, Automation and Systems (ICCAS), pp. 497-500.

Shinskey, F. G. (1996). Process control systems-application, design, and tuning. McGraw-Hill Inc, 4th ed., New York.

Tulleken, H. J. A. F. (1990). Generalized binary noise test-signal concept for improved identification-experiment design. Automatica, Vol. 26, $\mathrm{N}^{\circ} 1$, pp. 37-49. DOI: 10.1016/0005-1098(90)90156-C

Zhu, Y. (2001). Multivariable System Identification for Process Control. Elsevier Science, Oxford. 